\title{
Wireless Sensor Network Location Algorithm Based on Intelligent Estimation
}

\author{
Yu Jiang \\ Public Security Fire Force College, Kunming,650217, China
}

Keywords: Wireless; sensor; network positioning

Abstract: Wireless sensor networks are widely used in environment, transportation, military, aviation, medical and health, with many nodes and large network scale. How to use the intelligent estimation optimization algorithm to design a positioning mechanism to meet the requirements of low cost, low energy consumption, low dependence, strong robustness and high adaptability, which is the research and development target of modern large-scale wireless sensor networks.

\section{Introduction}

With the rapid development of sensor technology, embedded technology and communication and semiconductor technology, it has entered the post-PC era by the Pc era and the network era. Smaller, more convenient low-power computing devices break through the design patterns of traditional desktop and high-performance servers. The sensor network is one of the new information acquisition and processing technologies that are highly integrated with the above technologies. At present, wireless sensor networks have become an active research branch in the field of computer science, which has attracted great attention from academia and industry and is considered to be one of the technologies that will have a great influence on the 21 st century ${ }^{[1]}$. WSN consists of sensor nodes with sensing, limited data processing capability and short-range wireless communication functions and gateway nodes with strong data processing and long-distance wireless communication functions in military defense, environmental monitoring, biomedical, disaster relief and commercial applications. The field has broad application prospects. Wireless sensor networks can be divided into active and reactive types. Reactive sensor networks are better suited for time-sensitive applications because active wireless sensor networks continuously monitor surrounding material phenomena and transmit monitoring data at a constant rate, while reactive wireless sensor networks only change when the observed variable is abrupt. Transfer data.

\section{Composition of wireless sensor network nodes}

In recent years, advances in technologies such as wireless communications, microelectronics and embedded computing have driven the development of low-cost, low-power and multi-sensor nodes that enable information acquisition, data processing and short-range integration in a tiny volume. Various functions such as wireless communication. The wireless sensor network node is composed of a data acquisition module (sensor, A/D converter), a data processing and control module 
(microprocessor, memory), a communication module (wireless transceiver) and a power supply module (battery, energy converter) and the like, in which a positioning module, a mobile device and the like can be attached. Node location algorithms are a critical issue in wireless sensor networks and are the basis for a variety of applications. A high-precision, distributed, low-complexity and fault-tolerant positioning algorithm is needed to obtain the location of all unknown nodes. This can improve the performance of wireless sensor networks and on the other hand, it can reduce costs and benefit its Large-scale application ${ }^{[2]}$.

The intelligent estimation optimization algorithm is generally to solve the optimization problem. The optimization problem can be divided into a function optimization problem of solving a function in which a function value is the smallest and a combination optimization problem in which a solution is found in an solution space and the objective function value is minimized. Typical combinatorial optimization problems are: traveling salesman problem, processing scheduling problem, 0-1 knapsack problem and packing problem. There are many optimization algorithms. The classical algorithms include: linear programming, dynamic programming, etc.; improved local search algorithms include hill climbing method, steepest descent method, etc. The simulated annealing, genetic algorithm and search introduced in this paper are called guiding search methods. In neural networks, chaotic search is a dynamic evolution method of systems. The neighborhood function is often mentioned in the optimization idea and its role is to indicate how to obtain a new (group) solution from the current solution. The specific implementation method should be determined according to the analysis of specific problems. In general, local search is based on greedy thinking using the neighborhood function to search. If you find a solution that is better than the existing value, the former is discarded and the latter is taken. However, it generally only gets a "local minimum solution", that is to say, it is possible that the rabbit climbed "Teng Tai Shan and the Little World" but did not find Mount Everest. Simulated annealing, genetic algorithm, search, neural network, etc. have been improved from different angles and strategies and a better "global minimum solution" has been achieved. The development trend of wireless sensors is shown in Figure 1.

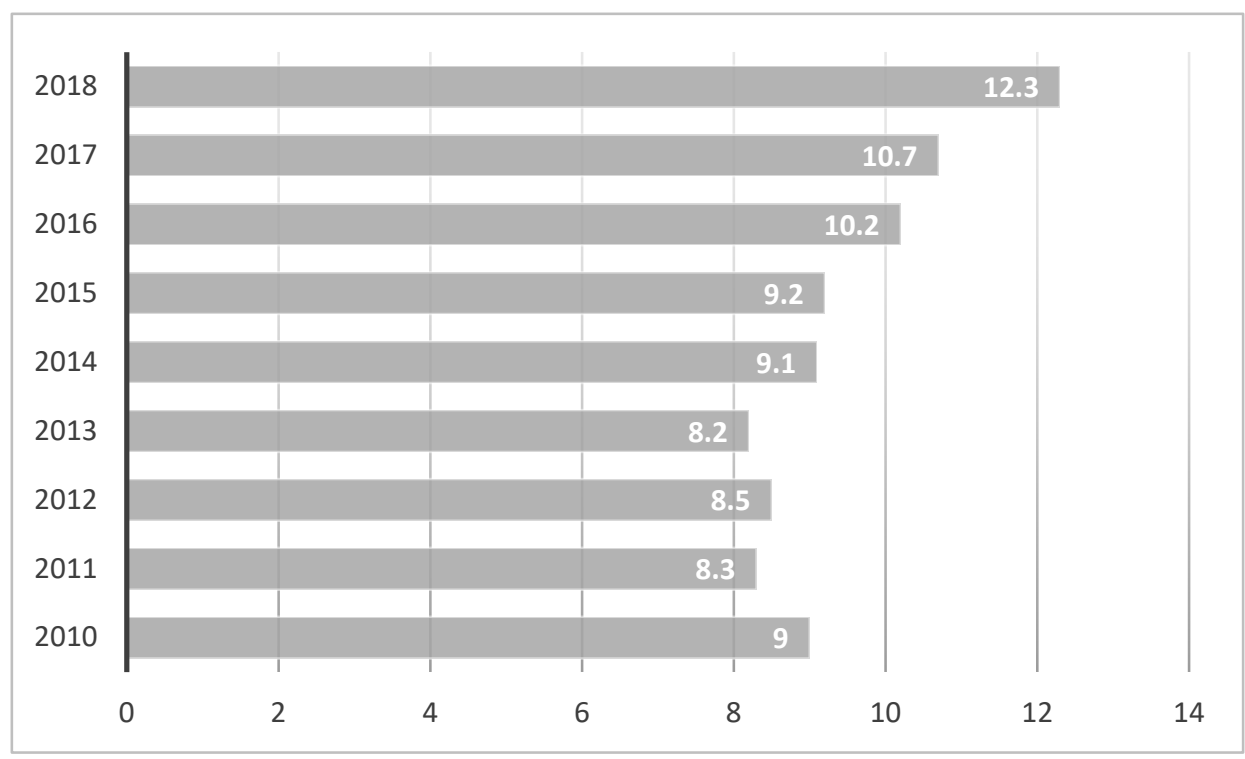

Figure 1: development trend of wireless sensors

\section{DV-hop positioning algorithm}

The positioning algorithm can be divided into two types of distance-related and 
distance-independent, depending on whether or not the distance is measured. Distance related information by measuring distance and other information requires high hardware and high cost. Limited by the wireless sensor network hardware facilities, the distance-independent mechanism is considered to be a cost-effective alternative to the cost-based method. The DV-hop algorithm was proposed by NIULESCUD in the Navigate project and is applicable to the ad-hoc network and has an accuracy of about $1 / 3$ of the range in the dense network. In a heterogeneous network, it contains sensor nodes and anchor nodes. The anchor node can not only perform single-level broadcasting, but also broadcast its location information to all nodes of the entire network. The node calculates its own position based on the received anchor node location, the number of hops of the anchor node and the average distance of each hop. The implementation of the algorithm is roughly divided into the following three stages ${ }^{[3]}$.

\subsection{Distance vector exchange phase}

In this phase, the DV-hop algorithm uses a mechanism similar to the classical distance vector routing algorithm, so that all nodes in the network know its hop count with each reference node. To this end, each node maintains a table $\{\mathrm{Xi}, \mathrm{Yi}, \mathrm{Hi}\}$, where $\mathrm{Xi}, \mathrm{Yi}$ are the coordinates of the reference node $\mathrm{i}$ and $\mathrm{Hi}$ is the number of hops from the node to the reference node. Initially, the reference node broadcasts a beacon (data packet) to the neighboring node containing its coordinates and hop count, with an initial value of one. After receiving the beacon, the neighbor node adds 1 to the hop and continues to broadcast to its neighbors (except the source direction), thus spreading to the entire network in a flooding manner. If a node receives multiple beacons from the same reference node, it indicates that it has multiple paths to the reference node. At this point, the node will retain the beacon with the smallest hop value, ignoring the other beacons, which ensures that the resulting hop value is its shortest path to the reference node. The positioning algorithm node distribution is shown in Figure 2.

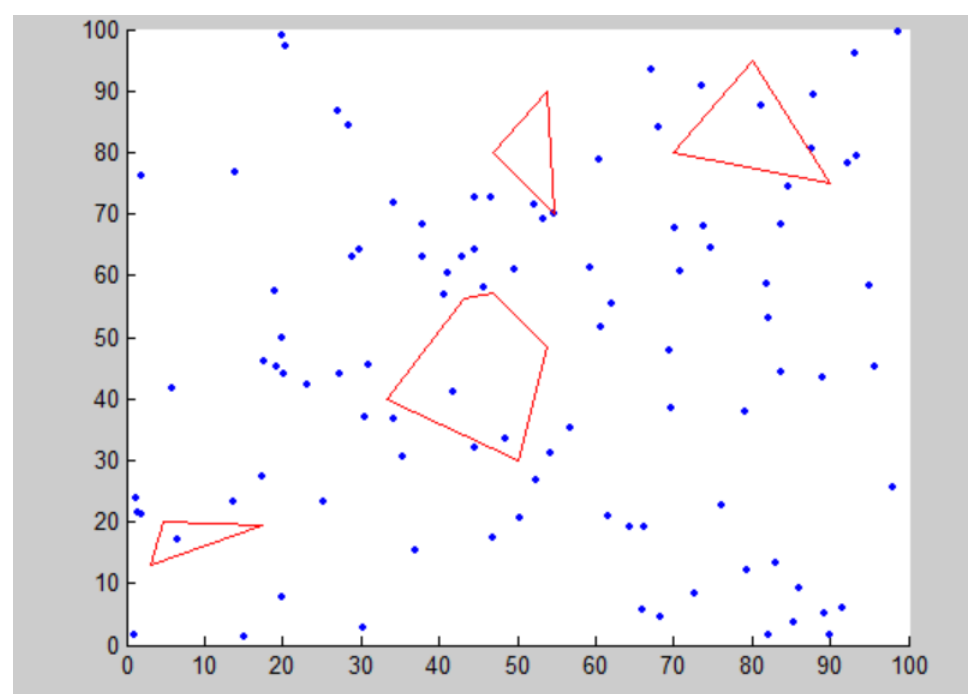

Figure 2: positioning algorithm node distribution

\subsection{Correction value calculation and broadcasting stage ${ }^{[4]}$}

In this phase, each reference node calculates the network average hop distance after obtaining other reference node positions and hops and then broadcasts it as a correction value to the network. Refer to the average hop distance of node $i$, which is the correction value. 


\subsection{Intelligent estimation stage}

Centralized algorithms and distributed algorithms. Centralized positioning means that the node transmits the information required for positioning to the central node and calculates the position of the central node row node; the distributed positioning is performed by the information exchange of the node and the positioning method assisted by the beacon node. Compared with centralized algorithms, distributed algorithms have the following characteristics: strong self-organization ability, independent of global infrastructure; robust, able to tolerate node failure and ranging error; energy saving requires less computation and communication overhead. Therefore, distributed algorithms are more suitable for large-scale sensor networks.

Distance related algorithms and distance independent algorithms. The distance-related algorithm calculates the node position using a positioning algorithm such as trilateration, triangulation, or maximum likelihood estimation by measuring the distance or angle information between the nodes. Without the ranging positioning algorithm, distance and angle information are not needed and the algorithm realizes node positioning according to information such as network connectivity ${ }^{[5]}$.

Distance related algorithm. This type of positioning algorithm is divided into two steps: the first step of ranging, using a specific ranging technique to measure the distance between the unknown node and the anchor node; the second step is to calculate, when the distance information obtained by the unknown node reaches a threshold, position of the unknown node is calculated using trilateration, triangulation, or maximum likelihood estimation. In the distance-independent positioning algorithm, the consumption of ranging accounts for the largest proportion in the positioning process, so the focus of research is on ranging technology.

\section{TEEN Routing Protocol Overview}

The TEEN protocol is real-time and can respond quickly to unexpected events. It is a routing strategy designed for reactive wireless sensor networks. TEEN uses the same multi-cluster structure and operation as LEACH. In the process of establishing the cluster, with the selection of the cluster head node, in addition to the data scheduling by the TDMA method, the cluster head broadcasts the hard threshold (HT) and soft threshold (ST) parameters of the data to the members of the cluster. The hard threshold is a threshold that cannot be exceeded by the detected data and the soft threshold specifies the range of variation of the detected data. During the steady phase of the cluster, the nodes constantly perceive their surroundings through sensors. When the node first detects the data arrival threshold, the transceiver is turned on for data transmission and the detected value is stored in the node internal variable SV. There are two conditions to be met when the node performs data transfer again:

(1) The current detected value is greater than the hard threshold;

(2) The difference between the current detected value and the SV is equal to or greater than the soft threshold. As long as the node sends data, the variable SV is set to the current detected value. In the process of cluster reconstruction, if the cluster head of the new round has been determined, the cluster head will reset and release the above two parameters.

The advantages of the TEEN protocol are:

1) The protocol is suitable for an application environment that requires real-time awareness;

2) By setting two parameters of hard threshold and soft threshold, TE

EN can greatly reduce the number of data transfers and is more energy efficient than the LEACH algorithm;

3) Since the soft threshold can be changed, the monitor can conveniently balance the two indicators of monitoring accuracy and system energy saving by setting different soft thresholds;

4) With the change of the cluster head, the user can reset the values of the two parameters as 
needed to control the number of data transmissions. The main disadvantage of ETEN is that it is not suitable for applications that require periodic data acquisition. This is because if a node in the network does not receive the relevant threshold, then the node will not communicate with the sink node and the user will not get any data from the network at all.

\section{Conclusion}

In this paper, the existing common wireless sensor network localization algorithms are compared and analyzed. So far, various positioning algorithms have certain technical defects. Even so, these existing algorithms have been promoted and developed for wireless sensing technology. The scientific and technological progress of the entire human society has made an indelible contribution.

\section{Acknowledgement}

Fund Project: Fire Services College School-level scientific research project, No.2018004

\section{References}

[1] Cheng Wu,Yiming Wang,Zhijie Yin. Energy-efficiency opportunistic spectrum allocation in cognitive wireless sensor network[J]. EURASIP Journal on Wireless Communications and Networking,2018,2018(1).

[2] Yanhong Ge,Shubin Wang,Jinyu Ma. Optimization on TEEN routing protocol in cognitive wireless sensor network[J]. EURASIP Journal on Wireless Communications and Networking,2018,2018(1).

[3] Quyuan Wang,Songtao Guo,Jianji Hu,Yuanyuan Yang. Spectral partitioning and fuzzy C-means based clustering algorithm for big data wireless sensor networks[J]. EURASIP Journal on Wireless Communications and Networking,2018,2018(1).

[4] Xiaodong Ji,Zhihua Bao,Chen Xu. Power minimization for OFDM modulated two-way amplify-and-forward relay wireless sensor networks[J]. EURASIP Journal on Wireless Communications and Networking,2017,2017(1).

[5] Zhiping Kang,Hong Zeng,Haibo Hu,Qingyu Xiong,Guangyu Xu. Multi-objective optimized connectivity restoring of disjoint segments using mobile data collectors in wireless sensor network[J]. EURASIP Journal on Wireless Communications and Networking,2017,2017(1). 